\title{
Development and Experiments of a Bio-inspired Underwater Microrobot with 8 Legs
}

\author{
Shuxiang Guo ${ }^{1,2}$, Liwei Shi ${ }^{1}$ and Kinji Asaka ${ }^{3}$ \\ ${ }^{1}$ Faculty of Engineering, Kagawa University, 2217-20 Hayashi-cho, Takamatsu, Kagawa \\ ${ }^{2}$ Harbin Engineering University, 145 Nantong Street, Harbin, Heilongjiang \\ ${ }^{3}$ Kansai Research Institute, AIST, 1-8-31 Midorigaoka, Ikeda, Osaka 563 \\ 1,3 Japan \\ ${ }^{2}$ China
}

\section{Introduction}

In recent two decades, research of underwater microrobots developed at a high speed. They can be widely applied in the field of underwater monitoring operations including pollution detection, video mapping, and exploration of unstructured underwater environments. Based on the underwater monitoring, this kind of microrobot is of great interest for cleaning the micro pipeline in the radiate area, getting samples from the seabed for archeology or mining, and so on (Kim et al., 2005; Behkam \& Sitti, 2006; McGovern et al., 2008). For example, some underwater robots with screw propellers have been developed. However, the electromagnetic structure of traditional motors is difficult to shrink. So, motors are rarely found in this sort of application (Zhang et al., 2006a; Wang et al., 2008), and special actuator materials are used instead. As a result, many kinds of smart materials, such as ionic polymer metal composite (IPMC), piezoelectric elements, pneumatic actuator, shape memory alloy, which can be used as artificial muscles, have been reported (Heo, 2007; Park et al., 2007). Although problems such as electrical leakage, water safety, physical bulk, and high stiffness persist in real applications, these smart materials have been widely used as actuators to develop new type of microrobot.

Ionic polymer metal composite (IPMC) is an innovative material made of an ionic polymer membrane chemically plated with gold electrodes on both sides. Its actuation characteristics, such as suitable response time, high bending deformation and long life, have significant potential for the propulsion of underwater microrobots. Flexible IPMC propulsion blades operating at low driving voltages provide many new possibilities for underwater locomotion applications (Lee \& Kim, 2006; Nakadoi \& Yamakita, 2006; Dogruer et al., 2007; Punning et al., 2007; Liu et al., 2008). They have been widely used on soft robotic actuators such as artificial muscles, as well as on dynamic sensors (Guo et al., 2008b; Ye et al., 2008). Now, many kinds of underwater microrobots have been developed using IPMC actuators as artificial muscles to propel the robots back and forth. They are widely used in swimming microrobots as oscillating or undulating fins where fast response is required (Jung et al., 2003; Guo et al., 2004; Kamamichi, 2006; Guo et al., 2007; Brunetto et al., 2008). IPMC actuators are also used for underwater bipedal walking microrobots (Kamamichi et al., 2003; Guo et al., 2006; Yim et al., 2007), and a kind of ion-conducting polymer gel film microleg 
with two DOF has been developed (Zhang et al., 2006c). In addition, a six-legged insectinspired underwater walking microrobot has also been described (Zhang et al., 2006d). For the six-legged one, the driving forces on the left and right sides are different because of the device's asymmetry. It has difficulty maintaining a straight path when walking forward. Because its centre of gravity and centre of rotation are not the same, it cannot be positioned precisely, and it consumes a lot of energy while rotating. In addition, the six-legged device cannot perform the movements required to dive or surface. With the aim of creating a compact structure with efficient and precise locomotion, we first developed a biomimetic underwater microrobot with eight IPMC actuators as legs to provide better performance in walking and rotating.

The remainder of this paper consists of five parts. Firstly, we described the design of the biomimetic leg shown in Figure 2. Secondly, we proposed a new type of microrobot with three DOF shown in Figure 4, including its walking, rotating, and floating mechanisms. Thirdly, we evaluated the mechanical behavior of the IPMC actuator, analyzed the forces applied to the four driving legs and simulated the walking speed. Fourthly, we developed the prototype microrobot and carried out experiments to measure its walking and rotating speeds on underwater flat. And the last is our conclusions.

\section{The biomimetic locomotion}

Each leg of a stick insect is composed of a coxa, a femur, a tibiae, and a tarsus. The tarsus is also called foot, and does not contribute to the movements. The coxa offers the foot one DOF motion in the direction of movement. The femur and the tibiae offer the foot a 2-DOF motion to enable it to find a reliable foothold together in the swing-search phase and touch the ground and support the body while moving in the stance phase, as shown in Figure 1. (Zhang et al., 2006a; Zhang et al., 2006b; Zhang et al., 2006d)

Based on the walking motion of the stick insect, a stick insect inspired biomimetic locomotion prototype using two IPMC actuators is developed, as shown in Figure 2. The actuator in vertical direction is called the driver. The actuator in the horizontal direction is called the supporter. The free end of the driver is the foot. The driver and supporter are controlled by two channels of square wave signals, with the same frequency. The driver and supporter can bend along Tra.2 and Tra.1 respectively. The phase of supporter is 90 degrees delayed than that of driver, so that driver and supporter can cooperate with each other in clawing motion as shown in Figure 3, where the swing-search phase is from (a) to (d) and the stance phase is from (d) to (e) (Zhang et al., 2006c; Zhang et al., 2006d).

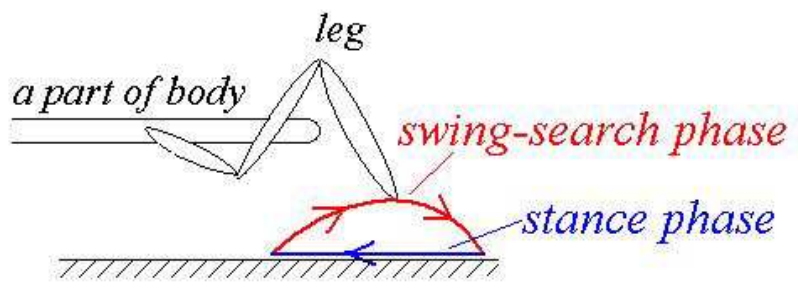

Fig. 1. Two phases in stick insect walking 


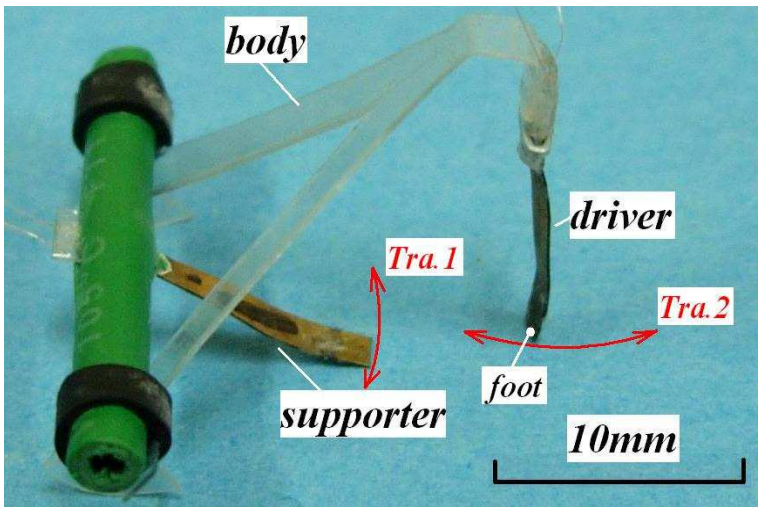

Fig. 2. The prototype of the biomimetic locomotion

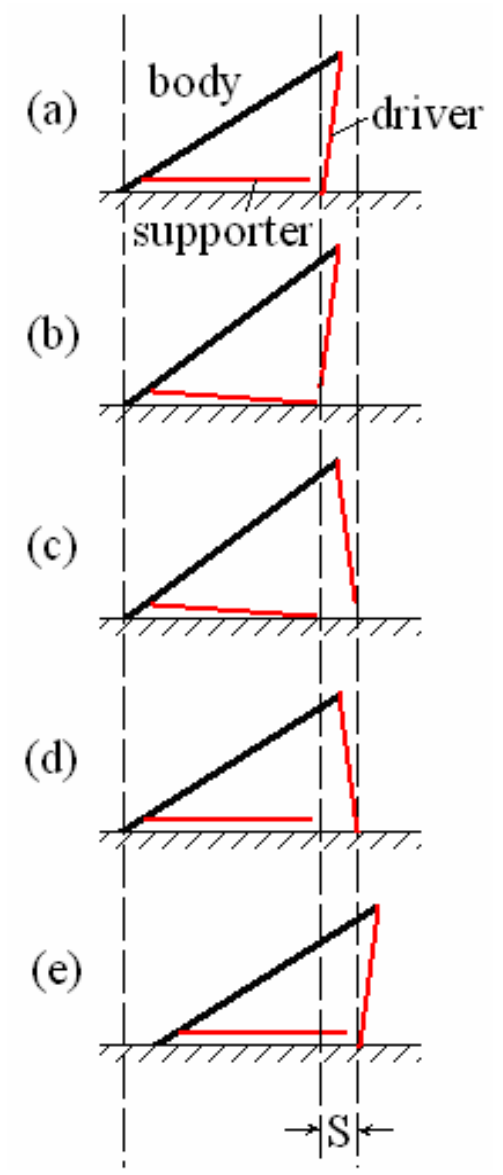

Fig. 3. One step cycle of the biomimetic locomotion 


\section{Proposed Eight-legged Microrobot}

\subsection{Proposed Eight-legged Microrobot Structure}

Using this locomotion, we had previously developed several microrobots (Zhang et al., 2006a; Zhang et al., 2006d). As shown in Figure 4, both of them have six IPMC actuators on plastic film body, which are divided into two groups, the drivers are from A to $C$, and the supporters are the others.

Walker- 1 can not only walk in 3 straight lines, but also rotate around its symmetric axis. Its disadvantage is that its moving motion is inefficient because one driver always resists forward moving limited by its structure(Zhang et al., 2006d). For the Walker-2, the drivers and the supporters are on both sides of a rectangle film body. However, the driving forces on the left and right sides are different because of the device's asymmetry. So, it has difficulty maintaining a straight path when walking forward. Because its centre of gravity and centre of rotation are not the same, it cannot be positioned precisely, and it consumes a lot of energy while rotating. In addition, it cannot perform the movements required to dive or surface with a stable gesture for its asymmetry.

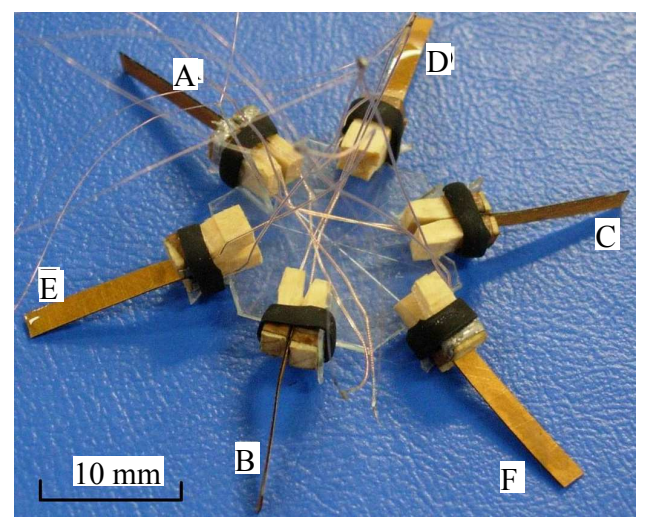

Walker-1

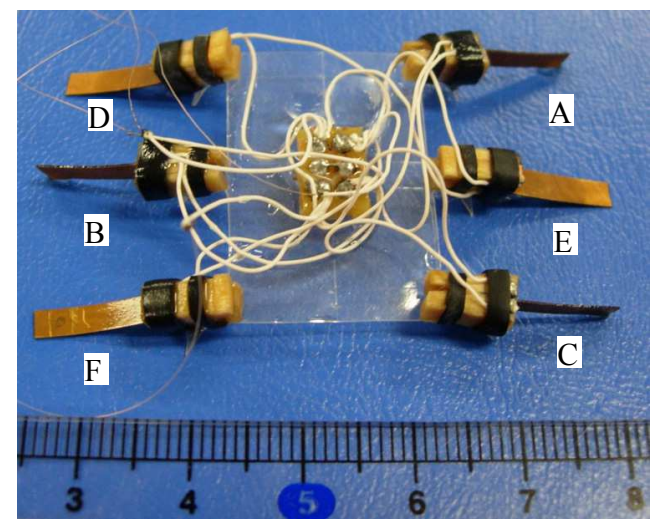

Walker-2

Fig. 4. Photos of previously developed six-legged biomimetic microrobots

To inherit the advantages of previously developed microrobots and overcome their disadvantages, we proposed a new biomimetic microrobot with a centrosymmetric structure around which the eight legs are symmetrically distributed, as shown in Figure 5 and Figure 6. It is $33 \mathrm{~mm}$ long, $56 \mathrm{~mm}$ wide, and $9 \mathrm{~mm}$ high. It has eight IPMC actuators designated A through $\mathrm{H}$. Actuators A, B, C, and D are the drivers, and their bending directions are shown in Figure 7. The other four actuators are supporters. The actuators are all $11 \mathrm{~mm}$ long, $3 \mathrm{~mm}$ wide, and $0.2 \mathrm{~mm}$ thick. The distance between two adjacent drivers or between a driver and a supporter is $10 \mathrm{~mm}$ (Guo et al. 2008a; Guo et al. 2009).

The biomimetic microrobot is capable of walking, rotating, and floating. Table 1 lists the control strategies for crawling. 


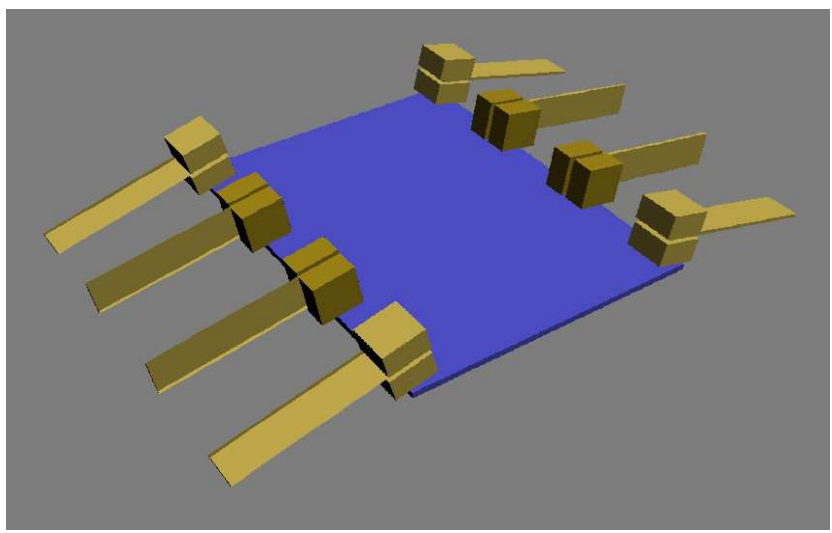

Fig. 5. Proposed eight-legged biomimetic microrobot

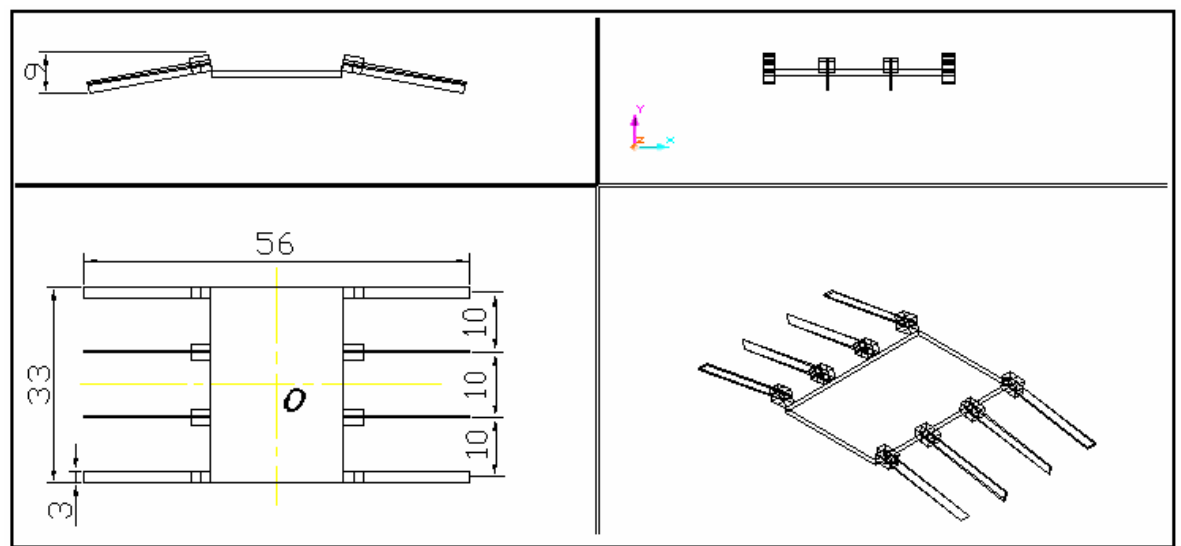

Fig. 6. Proposed microrobot structure

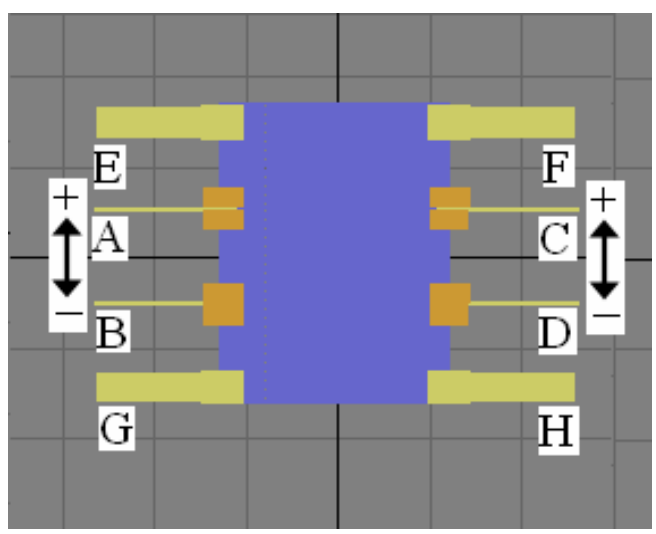

Fig. 7. Bending directions for the four drivers 


\begin{tabular}{c|c|c|c|c}
\hline \hline Motions & A & B & C & D \\
\hline Walking forward & + & + & + & + \\
\hline Walking backward & - & - & - & - \\
\hline Rotating in clockwise & + & + & - & - \\
\hline Rotating in counter clockwise & - & - & + & + \\
\hline \hline
\end{tabular}

A, B, C, and D stand for the drivers, as shown in Fig. 7. "+" and "_" mean the drivers bending forward and backward, respectively.

Table 1. Control strategies for crawling locomotion

\subsection{Crawling motion mechanism}

The drivers and supporters are driven at the same oscillating frequency. When crawling, the phase of the supporters lags that of the four drivers by $90^{\circ}$. Each step cycle of the walking motion can be separated into four periods as shown in Figure 8:

1. From $d$ to a, the supporters lift the body up, and the drivers are off the ground.

2. From $a$ to $b$, the drivers bend forward.

3. From $b$ to $c$, the supporters bend upward far enough so that they are off the ground, while the drivers in contact with the ground support the body.

4. From $\mathrm{c}$ to $\mathrm{d}$, the drivers bend backward in the propulsion stroke, and the body is pushed forward (Guo et al., 2008a).

During periods (1), (2), and (3), the drivers are off the ground and move to another foothold point with the help of the supporters. In period (4), the drivers push the body forward.

The walking speed is determined by the displacement of the drivers and the frequency of the control signal. Because the four drivers are distributed symmetrically on both sides of the structure and they have the same size and mass, the four drivers support equivalent loads and drag forces. Therefore, the four drivers experience the same displacement for the same applied input voltage. The deflections of all the drivers are equal, and they experience the same displacement in each step. The walking speeds on each side of the structure are the same. Suppose that the displacement of the actuator without a payload is $d_{0}$, and the microrobot can move forward with a displacement of $d$ in one step cycle. Because of the payload or water resistance, a decrease $\Delta d$ in the displacement of the drivers cannot be ignored. Equation (1) describes the relationship between $d_{0}$ and $d$, and Eq. (2) describes the walking speed, where $v$ is the average speed and $f$ is the frequency of the input signal.

$$
\begin{gathered}
d=d_{0}-\Delta d \\
v=\left(d_{0}-\Delta d\right) \times f
\end{gathered}
$$

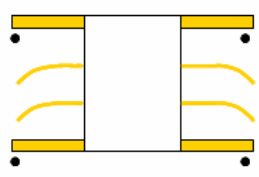

a

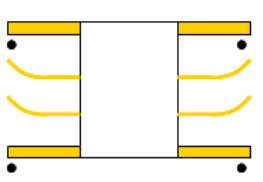

b

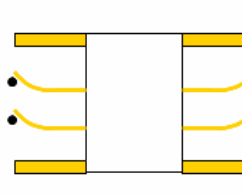

c

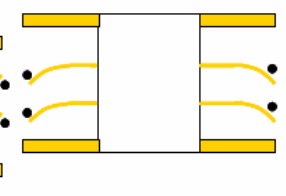

d

Fig. 8. One step cycle of walking forward. The $\bullet$ marks indicate which legs contact the ground. 


\subsection{Rotating motion mechanism}

Figure 9 shows the example of counter-clockwise rotation. From c to d, the drivers push the body to rotate. In the other periods, the drivers are pushed up by the supporters to prepare for the next stroke (Guo et al., 2008b).

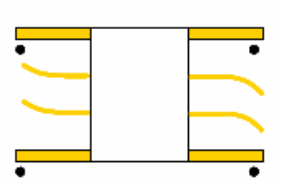

a
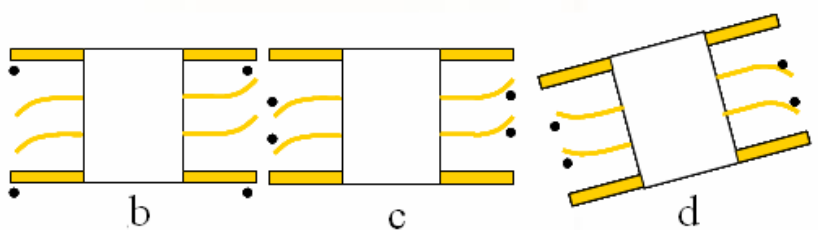

Fig. 9. One step cycle of the rotating motion. The $\bullet$ marks indicate which legs contact the ground.

The speed of the rotating motion is determined by the angle of the driver in one cycle and the frequency of the step, as shown in Figure 10 (a). The device can rotate by an angle of $\theta$ in one step cycle, as shown in Eq. (3).

$$
\theta=\frac{L}{R}=\frac{2 L}{D}
$$

Where $L$ is the length of the rotating arc, $R$ is the radius of the rotating rotundity, and $D$ is the diameter, as shown in Figure 10 (a) and Figure 10 (c). The diameter $D$ could be calculated using Eq. (4).

$$
D=\sqrt{(10+d)^{2}+(56-2 \Delta l)^{2}}
$$

Where $d$ is the displacement of the driver in the stance phase and $\Delta l$ stands for the displacement decrease of the IPMC actuator, as shown in Eq. (5).

$$
\Delta l=l-l_{s}
$$

Where $l$ is the length of IPMC actuator, $l$ and $l s$ are shown in Figure 10 (b). Based on the geometry theorem, we can get the Eq. (6).

$$
|\mathrm{AN}| \times|\mathrm{BN}|=|\mathrm{CN}| \times|\mathrm{DN}|
$$

From the Figure $10(b), r$ is the bending radius of the IPMC actuator, $|A N|=|B N|=l s$, $|\mathrm{CN}|=d / 2,|\mathrm{DN}|=|2 r-d / 2|$, so $l s$ can be calculated as shown in Eq. (7).

$$
l_{s}=\sqrt{\frac{d}{2} \times\left|2 r-\frac{d}{2}\right|}
$$

According to Eq. (3), Eq. (4), Eq. (5), and Eq. (7), the device can rotate by an angle of $\theta$ in one step cycle, as shown in Eq. (8).

$$
\theta=\frac{L}{R}=\frac{2 L}{D}=\frac{2 L}{\sqrt{(10+d)^{2}+\left[56-2\left(l-\sqrt{\frac{d}{2} \cdot\left|2 r-\frac{d}{2}\right|}\right)\right]^{2}}}
$$


Equation (9) gives the rotating speed. Here, because the rotating arc $L \approx d$, we can approximate $L$ by $d$ in Eq. (9). The $\omega$ and $f$ are the rotating speed and the frequency, respectively. Figure 10 (a) shows that the structure's centre of rotation is at point $O$, which is also the structure's centre of symmetry. Because the eight legs are symmetrically distributed around the centre of symmetry of the centrosymmetric structure and because the eight legs have the same size and mass, the centre of symmetry is also the centre of gravity. Thus, the centre of rotation of the microrobot is its centre of gravity. In addition, because the oscillating directions of the four drivers are close to perpendicular to the radius of rotation, the eight-legged device has a larger torsion angle than did the previous six-legged version.

$$
\omega=\theta^{*} f=\frac{2\left(d_{0}-\Delta d\right)}{\sqrt{(10+d)^{2}+\left[56-2\left(l-\sqrt{\frac{d}{2} \cdot\left|2 r-\frac{d}{2}\right|}\right)\right]^{2}}} f
$$

\subsection{Floating motion mechanism}

The water around the surface of the IPMC actuators is electrolysed by decreasing the frequency of the applied voltage to $0.3 \mathrm{~Hz}$. The buoyancy of the microrobot can be controlled by the resulting change in volume to make it float upwards, remain neutrally buoyant, or sink downwards. Table 2 lists the control strategies for the floating motion.

\begin{tabular}{|c|c|}
\hline \hline Conditions & Floating motions \\
\hline$\rho g(V+\Delta V)<m g$ & sinking downward \\
\hline$\rho g(V+\Delta V)=m g$ & suspended \\
\hline$\rho g(V+\Delta V)>m g$ & floating upward \\
\hline \hline
\end{tabular}

$\rho$ is the density of water, $g$ is the acceleration of gravity, $V$ is the volume of the microrobot, $\Delta V$ is the volume of the bubbles, and $m g$ is the weight of the microrobot.

Table 2. Control strategies of floating locomotion

\section{Theoretical dynamic performance analysis}

\subsection{Tip displacements of IPMC without payloads}

Figure 8 shows one step cycle for forward motion. The microrobot can move a distance of $d$ during one step cycle. We measured the displacement of one IPMC actuator by applying different signals to simulate the theoretical crawling speed of the microrobot as shown in Figure 11. The IPMC actuator was $14 \mathrm{~mm}$ long, $3 \mathrm{~mm}$ wide, and $0.2 \mathrm{~mm}$ thick. The actuator was driven by a PC equipped with a digital-to-analogue converter card, and the deflection of the IPMC was measured by a laser displacement sensor. The laser sensor was used to translate the displacement to a voltage, and then the voltages were recorded and translated to the PC using an oscilloscope.

Because the actuator was designed for use in a water tank, the relationship between the tip distance and the laser senor voltage was different from that relationship in air. This means that the laser sensor must first be calibrated for use in water (Guo et al, 2009). Figure 12 
shows the tip displacements of the actuator recorded experimentally for different frequencies and voltages. These results show that the tip displacement decreased as the frequency increased. Therefore, the microrobot had a top walking speed, and the theoretical walking speed could be calculated using equation (2).

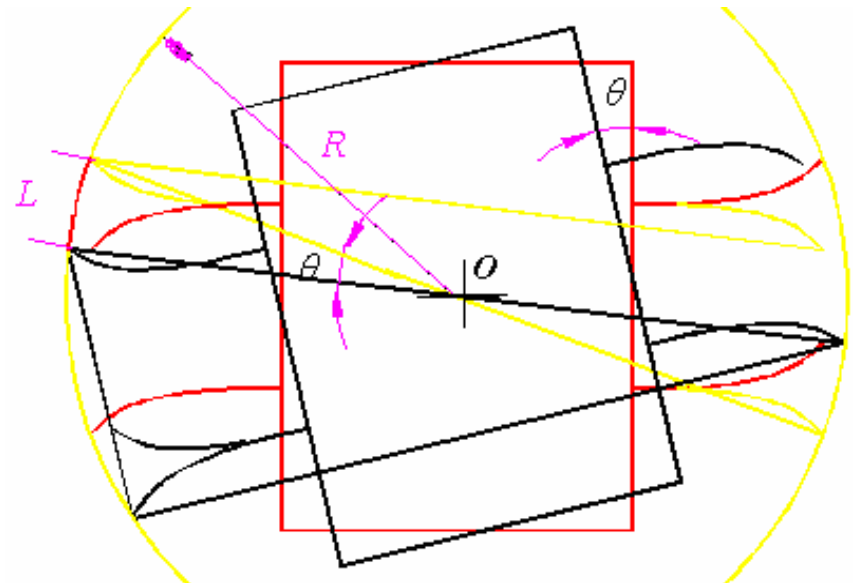

(a)

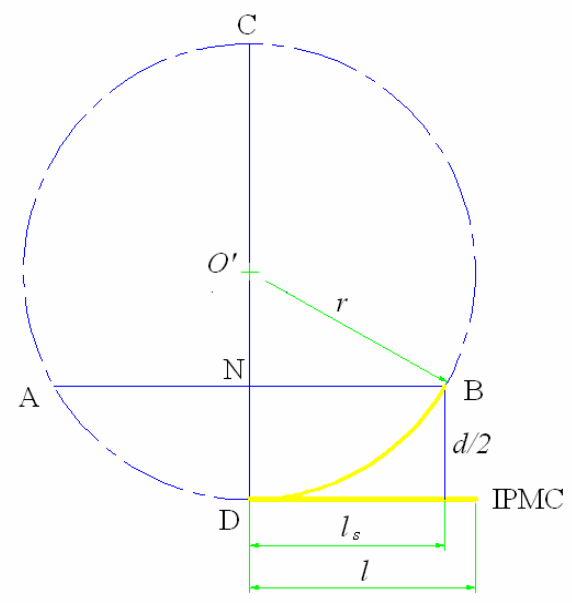

(b)

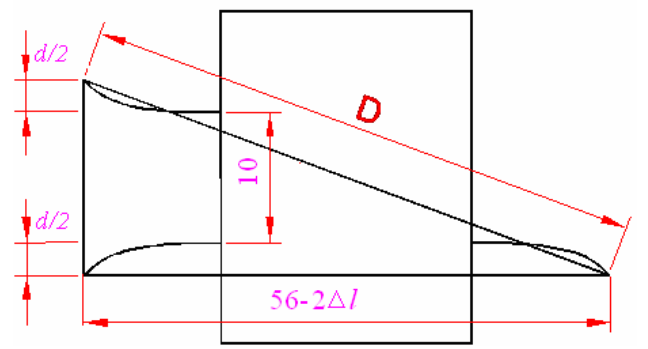

(c)

Fig. 10. Efficiency of the driver during rotating motion 


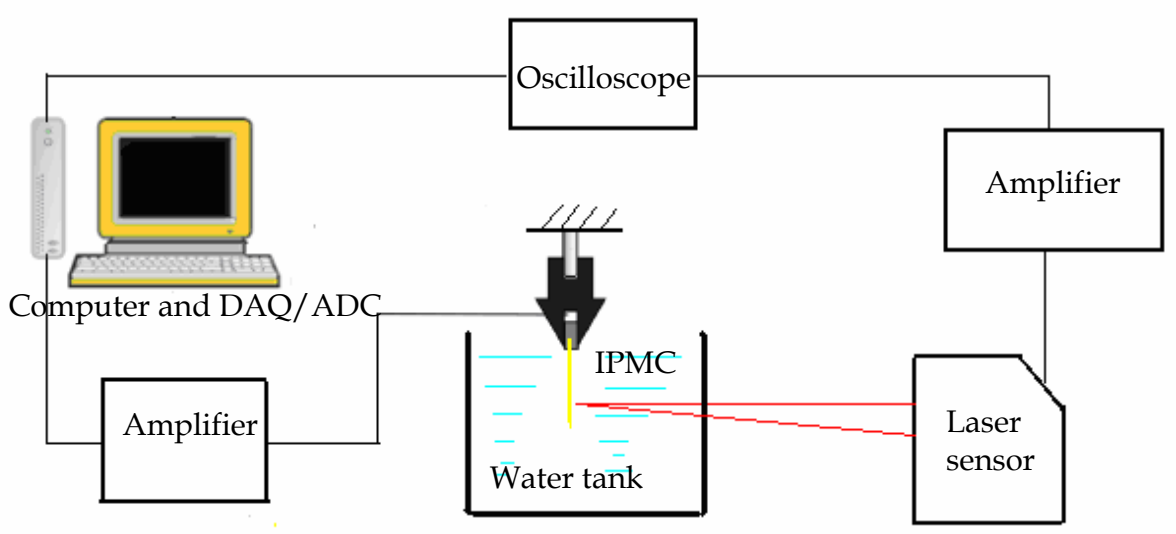

Fig. 11. Displacement measuring system

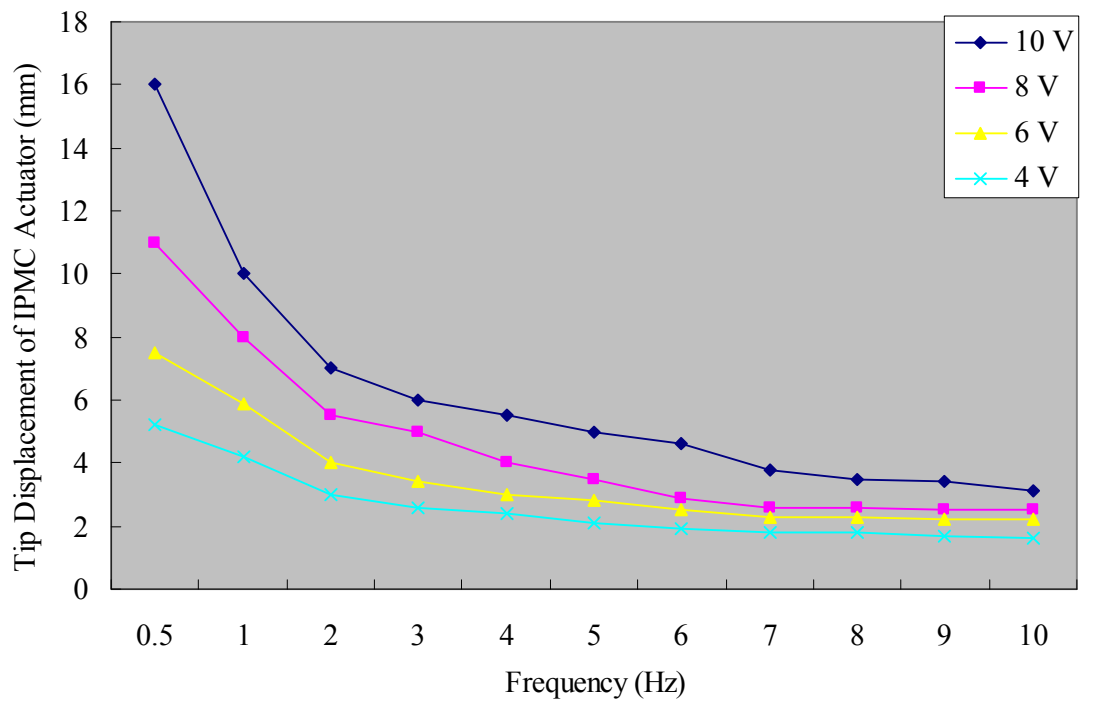

Fig. 12. Tip displacements $\left(d_{0}\right)$ of the IPMC actuator

\subsection{Equivalent beam modeling}

The IPMC beam actuator can be modelled as a supported cantilever beam as shown in Figure 13 (Cilingir et al., 2008; Pugal et al., 2008; Stoimenov et al., 2008; Mbemmo et al., 2008). When the microrobot crawling, the forces applied to one leg are shown in Figure 14, where $q$ is the surface tension of the IPMC actuator and $F$ is the resultant force of friction and water resistance to one leg. According to the cantilever beam theory, the relationship between the deformation curvature $1 / \rho(x)$ and mechanical moment $M$ is shown in the equation (10). 


$$
\frac{1}{\rho(x)}=\frac{M(x)}{E I}
$$

Where $E$ is the elastic modulus for IPMC in hydrated conditions and $I$ is the moment of inertia for the equivalent cantilever beam. Mechanical moment $M$, produced duo to IPMC bending is a function of applied forces.

Also, according to curvature equation (11) of deflection curve, we can get the equation (12).

$$
\begin{gathered}
\frac{1}{\rho(x)}=-\frac{\frac{d^{2} w}{d x^{2}}}{\left[1+\left(\frac{d w}{d x}\right)^{2}\right]^{3 / 2}} \\
\frac{\frac{d^{2} w}{d x^{2}}}{\left[1+\left(\frac{d w}{d x}\right)^{2}\right]^{3 / 2}}=-\frac{M(x)}{E I}
\end{gathered}
$$

For the small deflection, equation (12) is simplified as shown in equation (13), which is approximately expressed.

$$
\frac{d^{2} w}{d x^{2}}=-\frac{M(x)}{E I}
$$

The tip displacement generated by the surface tension $q$ in one direction, can be defined as $w_{q}$. So, the tip displacement in two directions $d_{0}=2 w_{q}$ can be calculated in equation (14).

$$
d_{0}=2 \times w_{q}=2 \times \frac{q x^{2}}{24}\left(-4 l x+6 l^{2}+x^{2}\right)=2 \times \frac{q l^{4}}{8 E I}=\frac{q l^{4}}{4 E I}
$$

The tip displacement generated by the resultant force $F$ in one direction, can be defined as $w_{\mathrm{F}}$. So, the tip displacement in two directions $\Delta d=2 w_{\mathrm{F}}$ can be calculated in equation (15). As a result, the resultant deflection $d$ can be obtained from equation (16).

$$
\begin{gathered}
\Delta d=2 \times w_{F}=2 \times\left(-\frac{F x^{3}}{6 E I}+\frac{F l x^{2}}{2 E I}\right)=\frac{2 F l^{3}}{3 E I} \\
d=d_{0}-\Delta d=2 \times\left(w_{q}-w_{F}\right)=d_{0}-\frac{2 F l^{3}}{3 E I}
\end{gathered}
$$

In order to calculate the $w_{\mathrm{F}}$, the IPMC bending stiffness $E I$ can be calculated from experiments and equation (17) respectively.

$$
I=\frac{b \cdot h^{3}}{12}
$$


The elastic modulus $E$ for IPMC in hydrated conditions is measured with the value of about $83 \mathrm{MPa}$ (Park et al., 2007). With cross dimension of $0.2 \times 3 \mathrm{~mm}$, the moment of inertia for the IPMC can be obtained about $2.0 \times 10^{-3} \mathrm{~mm}^{4}$. Then the bending stiffness is calculated as 166.0 $\mathrm{mN} \mathrm{mm}^{2}$. For one leg, the force $F$ is the friction, as shown in equation (18).

$$
F=\mu_{s} N
$$

Where $N$ is the positive pressure between the leg and the bottom of water tank, and $\mu_{\mathrm{s}}$ is the static friction coefficient. According to the materials of IPMC and the hard steel bottom, we choose $\mu_{\mathrm{s}}=0.30$ in our experiments. With the weight of $1.63 \mathrm{~g}$ in air and cubage of $0.79 \mathrm{~cm}^{3}$, the weight of the microrobot in water can be calculated as $0.84 \mathrm{~g}, \mathrm{~N}=0.84 \mathrm{~g}$. So, the force $F$ of one leg can be obtained about $8.232 \mathrm{mN}$. With the length of leg $l=11 \mathrm{~mm}$, the force $F=0.6175$ $\mathrm{mN}$, and the bending stiffness of the leg $E I=166.0 \mathrm{mN} \mathrm{mm}^{2}$, the displacement decreases of IPMC drivers $\Delta d$ can be evaluated as $3.301 \mathrm{~mm}$ and the walking speeds can be calculated by equation (2). The simulated results are shown in Figure 15.

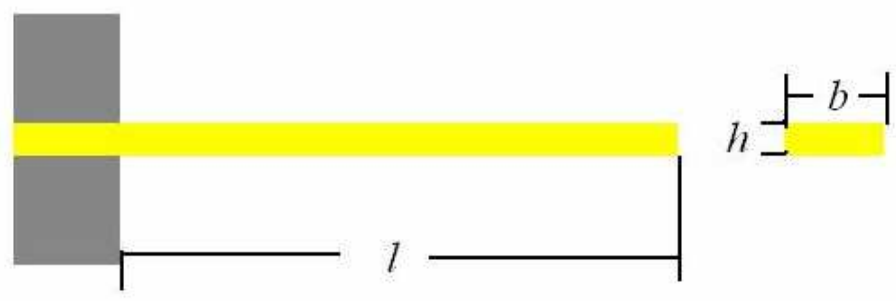

Fig. 13. Equivalent cantilever beam for an IPMC actuator

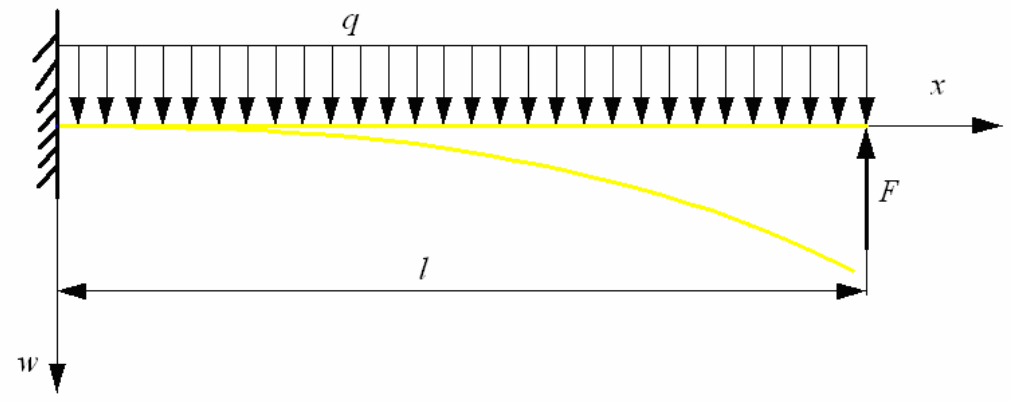

Fig. 14. Forces and deflection of IPMC actuator in $w$ direction 


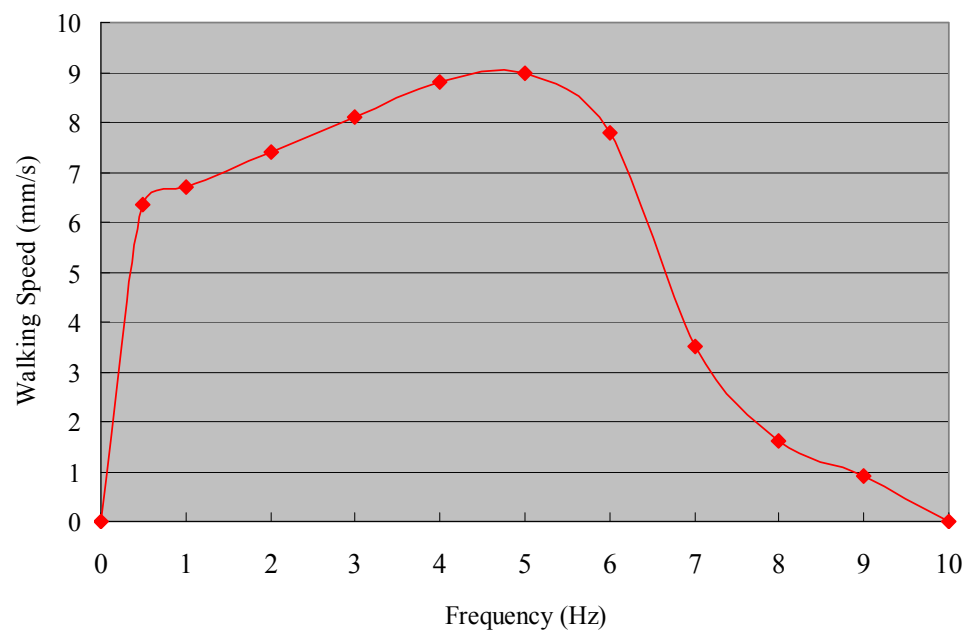

Fig. 15. Theoretical walking speed (10 V)

\section{Prototype microrobot and experiments}

\subsection{Prototype eight-legged microrobot}

Figure 16 shows the prototype of the eight-legged microrobot. It has eight actuators fixed on a film body with wood clips. The control signals are transmitted by enamel-covered wires $300 \mathrm{~mm}$ long with a copper diameter of $0.03 \mathrm{~mm}$. The wires are soft enough that the resistance can be ignored.

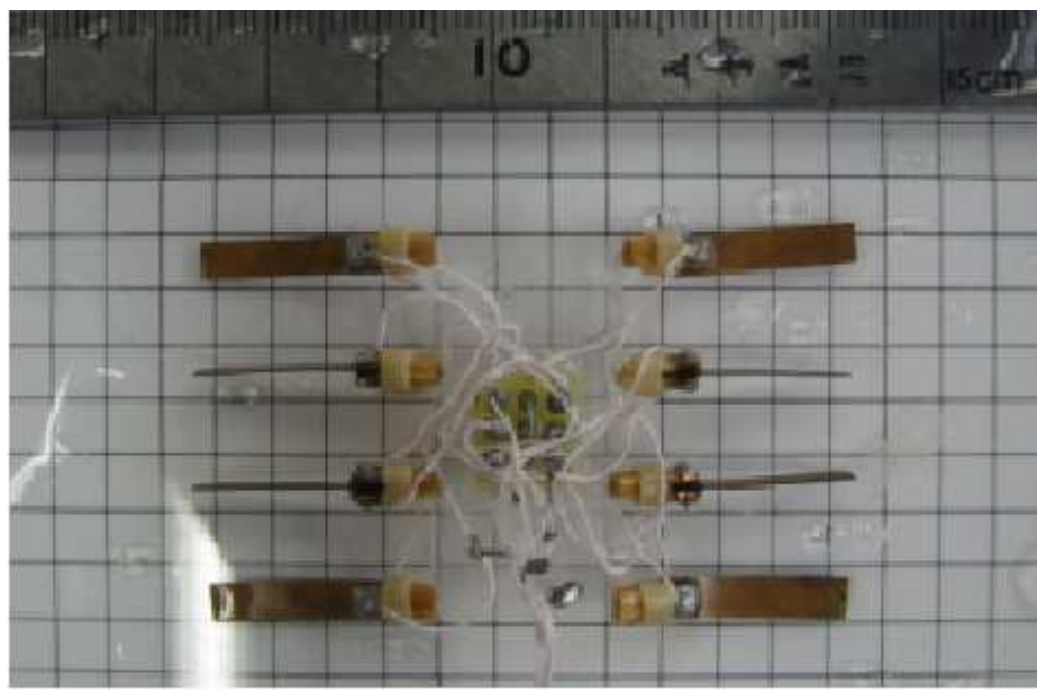

Fig. 16. Prototype eight-legged underwater microrobot 


\subsection{Walking experiment on underwater flat surface}

To evaluate walking locomotion, we carried out an experiment on an underwater plastic surface. We recorded the times required to walk a distance of $50 \mathrm{~mm}$ using different applied signal voltages and frequencies. The experiment was repeated 10 times for every set of control signals to determine the average speed on the flat surface. The experimental results described in Figure 17 show that the walking speed was nearly proportional to the input voltage, and a top speed of $8.3 \mathrm{~mm} / \mathrm{s}$ was obtained with a control signal of $10 \mathrm{~V}$ and $5 \mathrm{~Hz}$. We compared the experimental value with the theoretical value with a control signal of 10 $\mathrm{V}$, as shown in Figure 18. From the comparison, we could see that the experimental results approached the theoretical results very well. The displacement of the IPMC actuator would be less in real applications due to slippage and short response time at high frequencies. Therefore, some differences between the theoretical and experimental results still exist.

\subsection{Rotating experiment on underwater flat surface}

We also investigated the rotating motion on the same underwater plastic surface. We recorded the times for rotating through $90^{\circ}$ under the influence of different voltages and frequencies of the control signal, and calculated the average angular velocity for 10 repetitions of the same experiment. The experimental results described in Figure 19 show that the angular velocity was nearly proportional to the input voltage, and a top angular rotation speed of $11.86^{\circ}$ s was obtained for a voltage of $10 \mathrm{~V}$ and a frequency of $5 \mathrm{~Hz}$.

\subsection{Floating experiment}

To test floating locomotion, we set the frequency of the applied voltage to $0.15 \mathrm{~Hz}$ to electrolyse the water around the IPMC surface. When the input voltage was cut off while the microrobot was floating upward, the microrobot gradually stopped moving upward and then started to sink. The maximum upward floating speed was $4 \mathrm{~mm} / \mathrm{s}$ with a voltage of 10 $\mathrm{V}$ as shown in Figure 20.

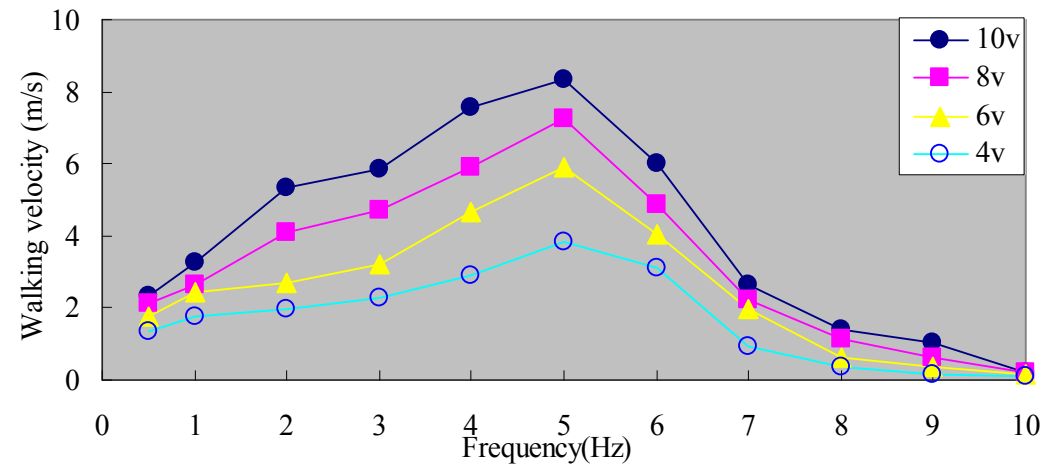

Fig. 17. Experimental walking speed results $(10 \mathrm{~V})$ 


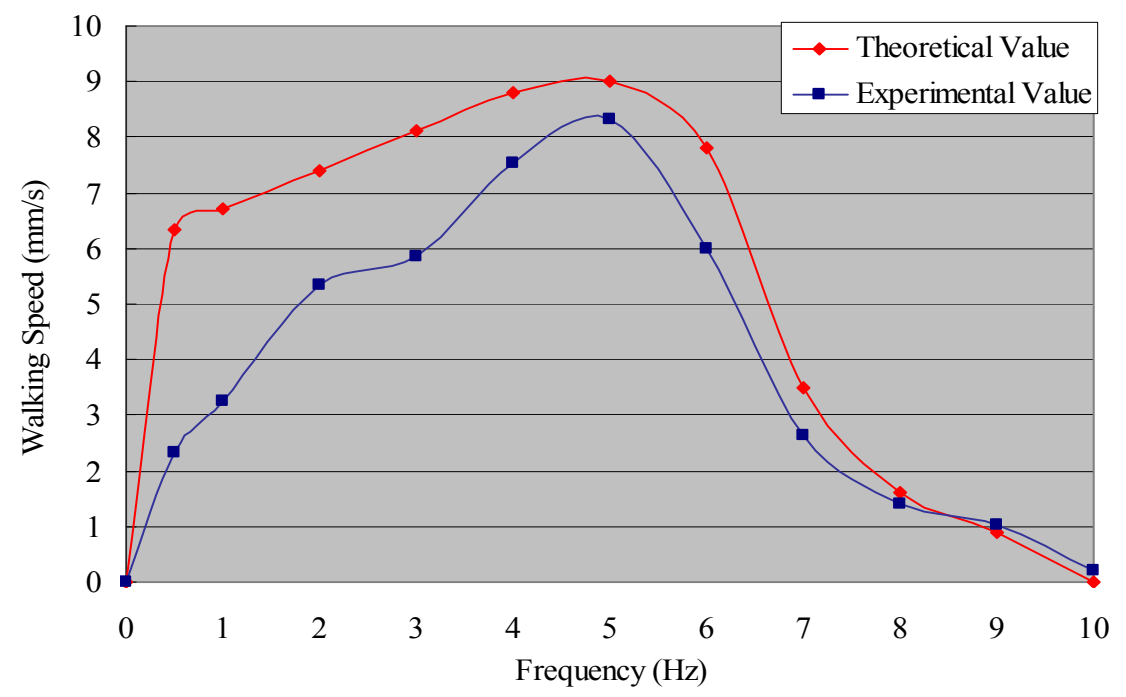

Fig. 18. Relationship between theoretical and experimental walking speeds (10 V)

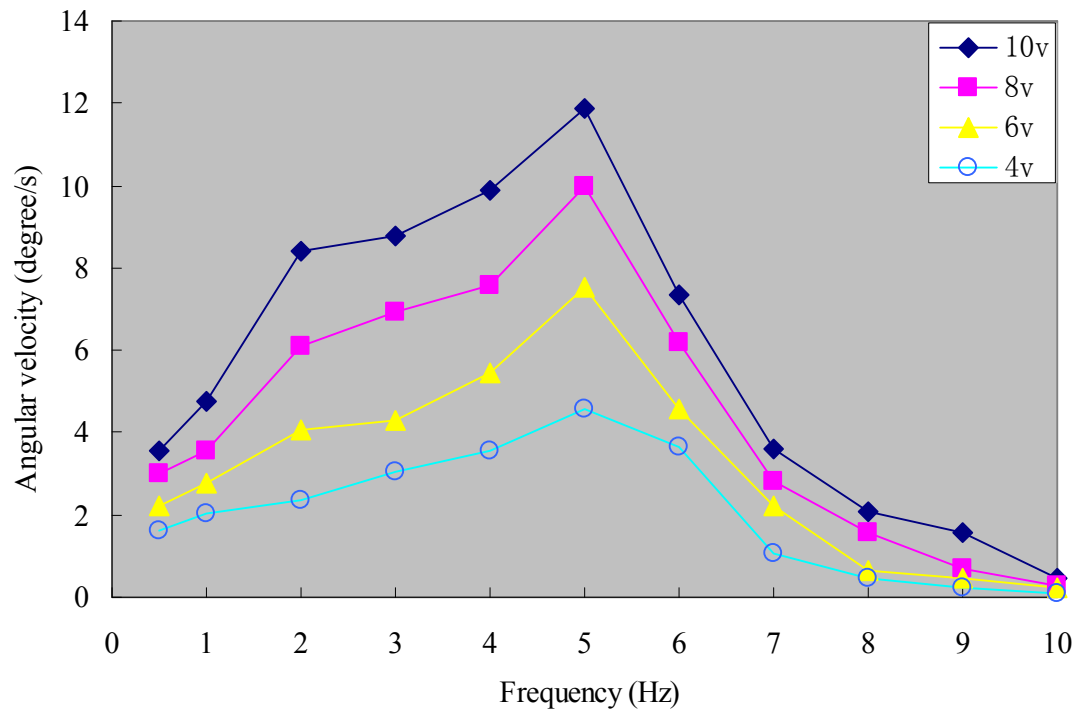

Fig. 19. Experimental angular velocity results during rotation 


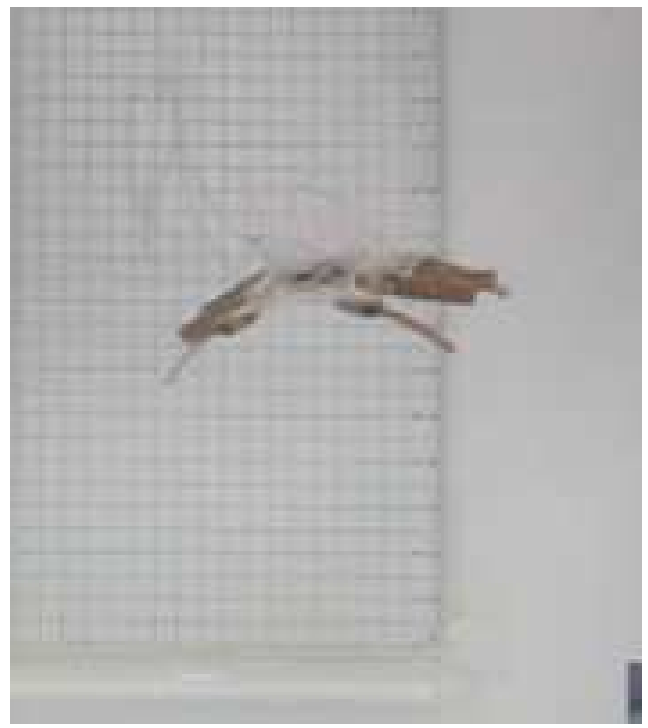

Fig. 20. Floating motion of the eight-legged microrobot

\section{Conclusions}

To resolve the problem of the asymmetry in previous six-legged microrobots, we proposed a new type of underwater microrobot with eight IPMC actuators distributed symmetrically around the microrobot's centre of symmetry. We evaluated the walking, rotating, and floating mechanisms of this proposed robot. Then, we evaluated the mechanical behavior of the IPMC actuator, analyzed the forces applied to the four driving legs and simulated the walking speed. We also constructed a prototype of the eight-legged microrobot and conducted experiments to measure its walking speed and angular velocity without payloads. Its walking and rotating speeds were faster than those of the previous six-legged version. We also made the microrobot dive and surface by electrolysing the water around the IPMC surface. Controlling the electrolysis process and thus the buoyancy of the microrobot was difficult, so the vertical motion of the device could not be controlled very well. In the following research, we developed a jellyfish-type microrobot to improve the floating motion.

\section{Acknowledgment}

This research is supported by Kagawa University Characteristic Prior Research Fund 2010.

\section{References}

Behkam, B. \& Sitti, M. (2006). Design methodology for biomimetic propulsion of miniature swimming robots, Journal of Dynamic Systems, Measurement, and Control, Vol.128, Issue 1, 2006, 36-43. 
Brunetto, P.; Fortuna, L.; Graziani, S. \& Strazzeri, S. (2008). A model of ionic polymer-metal composite actuators in underwater operations, Journal of Smart Material and Structures, 17, 2008, 025-029.

Cilingir, H.; Menceloglu, Y. \& Papila, M. (2008). The effect of IPMC parameters in electromechanical coefficient based on equivalent beam theory, Journal of Electroactive Polymer Actuators and Devices (EAPAD), Proc. of SPIE Vol. 6927, 2008, 69270L.

Dogruer, D.; Tiwari, R. \& Harvesters, K. (2007). Ionic Polymer Metal Composites as Energy Harvesters, Journal of Electroactive Polymer Actuators and Devices (EAPAD), Proc. of SPIE Vol. 6524, 2007, 65241C.

Guo, S.; Okuda, Y. \& Asaka, K. (2004). Development of a Novel Type of Underwater Micro Biped Robot with Multi DOF, Proceedings of the fourteenth of International offshore and polar engineering conference, vol. II, 2004, 284-289.

Guo, S.; Okuda, Y.; Zhang, W.; Ye, X. \& Asaka, K. (2006). The Development of a Hybrid Underwater Micro Biped Robot, Journal of Applied Bionics and Biomechanics, Vol.3, No.3, 2006, 143-150,.

Guo, S.; Shi, L. \& Asaka, K. (2008a). IPMC Actuator-based an Underwater Microrobot with 8 Legs, Proceedings of 2008 IEEE International Conference on Mechatronics and Automation, Japan, 2008, $551-556$.

Guo, S.; Shi, L. \& Asaka, K. (2008b). IPMC Actuator-Sensor based a Biomimetic Underwater Microrobot with 8 Legs, Proceedings of the IEEE International Conference on Automation and Logistics, China, 2008, 2495-2500.

Guo, S.; Shi, L.; Asaka, K. \& Li, L. (2009). Experiments and Characteristics Analysis of a Bioinspired Underwater Microrobot, Proceedings of the 2009 IEEE International Conference on Mechatronics and Automation, China, 2009, 3330-3335.

Guo, S.; Shi, L.; Ye, X. \& LI, L. (2007). A New Jellyfish Type of Underwater Microrobot, Proceedings of the 2007 IEEE International Conference on Mechatronics and Automation, China, 2007, 509-514.

Heo, S. (2007). Effect of an artificial caudal fin on the performance of a biomimetic fish robot propelled by piezoelectric actuators, Journal of Bionic Engineering, 4(3), 2007, 151 158.

Jung, J.; Kim, B.; Tak, Y. \& Park, J. (2003). Undulatory Tadpole Robot (TadRob) using ionic polymer metal composite (IPMC) actuator, Proceedings of the IEEE/RSJ Intl. Conference on Intelligent Robots and Systems, 2003, 2133-2138.

Kamamichi, N. (2006). A snake-like swimming robot using IPMC actuator/sensor, Proceedings of the 2006 IEEE International Conference on Robotics and Automation, 2006, 1812 - 1817.

Kamamichi, N.; Kaneda, Y.; Yamakita, M.; Asaka, K. \& Luo, Z. (2003). Biped Walking of Passive Dynamic Walker with IPMC Linear Actuator, SICE Annual Conference in Fukui, 2003, 212-217.

Kim, B.; Kim, D.; Jung a, J. \& J. Park. (2005). A biomimetic undulatory tadpole robot using ionic polymer-metal composite actuators, Journal of Smart Material and Structures, 14, 2005, 1579-1585.

Kim, S.; Lee, I. \& Kim, Y. (2007). Performance enhancement of IPMC actuator by plasma surface treatment, Journal of Smart Material and Structures, 16, 2007, N6-N11,. 
Lee, S. \& Kim, K. (2006). Muscle-like Linear Actuator Using an Ionic Polymer-Metal Composite and Its Actuation Characteristics, Journal of Smart Structures and Materials: Electroactive Polymer Actuators and Devices (EAPAD), Proc. of SPIE Vol. 6168, 2006, 616820.

Liu, S.; Lin, M. \& Zhang, Q. (2008). Extensional Ionomeric Polymer Conductor Composite Actuators with Ionic Liquids, Journal of Electroactive Polymer Actuators and Devices (EAPAD), Proc. of SPIE Vol. 6927, 2008, 69270H.

Mbemmo, E.; Chen, Z.; Shatara, S. \& Tan, X. (2008). Modeling of biomimetic robotic fish propelled by an ionic polymer-metal composite actuator, Proceedings of the IEEE International Conference on Robotics and Automation, 2008, 689-694.

McGovern, S. T.; Spinks, G. M.; Xi, B.; Alici, G.; Truong, V. \& Wallace, G. G. (2008). Fast bender actuators for fish-like aquatic robots, Proceedings of SPIE Vol. 6927, 2008, 69271L.

Nakadoi, H. \& Yamakita, M. (2006). Integrated Actuator-Sensor System on Patterned IPMC Film: Consideration of Electoric Interference, Proceedings of SI2006 International Conference, 2006.

Park, I. ; Kim, S.; Kim, D. \& Kin, K. (2007). The Mechanical Properties of Ionic PolymerMetal pomposites, Journal of Electroactive Polymer Actuators and Devices (EAPAD), Proc. of SPIE Vol. 6524, 2007, 65241R.

Pugal, D.; Kasemagi, H.; Kruusmaa, M. \& Aabloo, A. (2008). An Advanced Finite Element Model of IPMC, Journal of Electroactive Polymer Actuators and Devices (EAPAD), Proc. of SPIE Vol. 6927, 2008, 692711.

Punning, A.; Kruusmaa, M. \& Aabloo, A. (2007). Surface resistance experiments with IPMC sensors and actuators, Journal of Sensors and Actuators, A 133, 2007, 200-209.

Stoimenov, B.; Rossiter, J.; Mukai, T. \& Asaka, K. (2008). Frequency response of anisotropic ionic polymer metal composite (IPMC) transducers, Journal of Electroactive Polymer Actuators and Devices (EAPAD), Proc. of SPIE Vol. 6927, 2008, 69270K.

Wang, Z.; Hang, G.; Li, J.; Wang, Y. \& Xiao, K. (2008). A micro-robot fish with embedded SMA wire actuated flexible biomimetic fin, Journal of Sensors and Actuators, A 144, 2008, 354-360.

Ye, X.; Su, Y.; Guo, S. \& Wang, L. (2008). Design and Realization of a Remote Control Centimeter-Scale Robotic Fish, Proceedings of the 2008 IEEE/ASME International Conference on Advanced Intelligent Mechatronics, China, 2008, 25-30.

Yim, W.; Lee, J. \& Kim, K. (2007). An artificial muscle actuator for biomimetic underwater propulsors, Journal of Bioinspiration and Biomimetics. 2, 2007, S31-S41.

Zhang, W.; Guo, S. \& Asaka, K. (2006a). A New Type of Hybrid Fish-like Microrobot, International Journal of Automation and Computing, Vol.3, No.4, 2006, 358-365.

Zhang, W.; Guo, S. \& Asaka, K. (2006b). Characteristics Analysis of a Biomimetic Underwater Walking Microrobot, Proceedings of the 2006 IEEE International Conference on Robotics and Biomimetics, Kuming, China, 2006, 1600-1605.

Zhang, W.; Guo, S. \& Asaka, K. (2006c). Development of a novel type of an underwater microrobot with biomimetic locomotion, Journal of Applied Bionics and Biomechanics. Woodhead Publishing, Limited, Vol.3, No.3, 2006, 245-252.

Zhang, W.; Guo, S. \& Asaka, K. (2006d). Development of an underwater biomimetic microrobot with both compact structure and flexible locomotion, Journal of Microsystem Technologies, DOI 10.1007 : s00542-006-0294-9, 2006. 


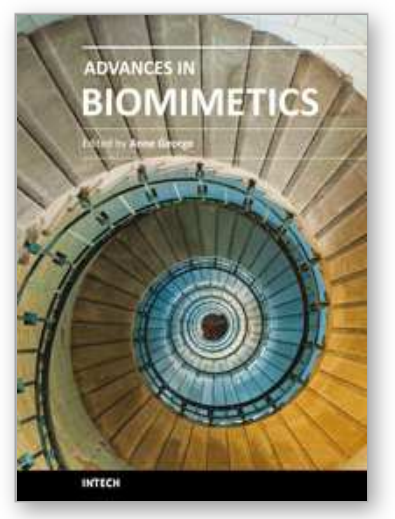

\author{
Advances in Biomimetics \\ Edited by Prof. Marko Cavrak
}

ISBN 978-953-307-191-6

Hard cover, 522 pages

Publisher InTech

Published online 26, April, 2011

Published in print edition April, 2011

The interaction between cells, tissues and biomaterial surfaces are the highlights of the book "Advances in Biomimetics". In this regard the effect of nanostructures and nanotopographies and their effect on the development of a new generation of biomaterials including advanced multifunctional scaffolds for tissue engineering are discussed. The 2 volumes contain articles that cover a wide spectrum of subject matter such as different aspects of the development of scaffolds and coatings with enhanced performance and bioactivity, including investigations of material surface-cell interactions.

\title{
How to reference
}

In order to correctly reference this scholarly work, feel free to copy and paste the following:

Shuxiang Guo, Liwei Shi and Kinji Asaka (2011). Development and Experiments of a Bio-inspired Underwater Microrobot with 8 Legs, Advances in Biomimetics, Prof. Marko Cavrak (Ed.), ISBN: 978-953-307-191-6, InTech, Available from: http://www.intechopen.com/books/advances-in-biomimetics/development-andexperiments-of-a-bio-inspired-underwater-microrobot-with-8-legs

\section{INTECH}

open science | open minds

\section{InTech Europe}

University Campus STeP Ri

Slavka Krautzeka 83/A

51000 Rijeka, Croatia

Phone: +385 (51) 770447

Fax: +385 (51) 686166

www.intechopen.com

\section{InTech China}

Unit 405, Office Block, Hotel Equatorial Shanghai

No.65, Yan An Road (West), Shanghai, 200040, China

中国上海市延安西路65号上海国际贵都大饭店办公楼 405 单元

Phone: +86-21-62489820

Fax: +86-21-62489821 
(C) 2011 The Author(s). Licensee IntechOpen. This chapter is distributed under the terms of the Creative Commons Attribution-NonCommercialShareAlike-3.0 License, which permits use, distribution and reproduction for non-commercial purposes, provided the original is properly cited and derivative works building on this content are distributed under the same license. 